\title{
The HIV cascade of care among serodiscordant couples in four high HIV prevalence settings in sub-Saharan Africa
}

\author{
M L Chihana, ${ }^{1,2}$ MSc; N Conan, ${ }^{2}$ MSc; T Ellman, ${ }^{3}$ BSc, MSc, MB ChB, MRCP; E Poulet, ${ }^{4}$ MSc; D B Garone, ${ }^{5}$ PhD; R Ortuno, ${ }^{6}$ MSc; \\ S Wanjala, ${ }^{7} \mathrm{MSc}$; C Masiku, ${ }^{8} \mathrm{MSc}$; J-F Etard, ${ }^{4,9} \mathrm{PhD}$; M-A Davies, ${ }^{1} \mathrm{MB}$ ChB, MMed, PhD, FC; D Maman, ${ }^{1,8} \mathrm{PhD}$ \\ ${ }^{1}$ School of Public Health and Family Medicine, Faculty of Health Sciences, University of Cape Town, South Africa \\ ${ }^{2}$ Epicentre, Cape Town, South Africa \\ ${ }^{3}$ Médecins Sans Frontières, Southern Africa Medical Unit (SAMU), Cape Town, South Africa \\ ${ }^{4}$ Epicentre, Paris, France \\ ${ }^{5}$ Médecins Sans Frontières, Brussels, Belgium \\ ${ }^{6}$ Médecins Sans Frontières, Blantyre, Malawi \\ ${ }^{7}$ Médecins Sans Frontières, Nairobi, Kenya \\ ${ }^{8}$ Médecins Sans Frontières, Lilongwe, Malawi \\ ${ }^{9}$ Montpellier University, TransVIHMI, Montpellier, France
}

Corresponding author: M L Chihana (menardchihana@yahoo.com)

Background. HIV-serodiscordant couples are at high risk of HIV transmission. In sub-Saharan Africa, HIV-serodiscordant couples contribute $\sim 30 \%$ of all new infections in the region.

Objectives. To quantify the prevalence of HIV-serodiscordant couples and evaluate steps of the HIV cascade of care among people living with HIV in serodiscordant relationships in four high-prevalence settings in sub-Saharan Africa.

Methods. Four HIV prevalence surveys were conducted: in Ndhiwa (Kenya) in 2012, in Chiradzulu (Malawi) in 2013, and in Gutu (Zimbabwe) and Nsanje (Malawi) in 2016. Eligible individuals aged 15 - 59 years were asked to participate in voluntary rapid HIV testing. Viral load and CD4 counts were measured on those who tested HIV-positive. A couple was defined as a man and a woman who reported being married or cohabiting and were living together in the same household.

Results. Among 4385 couples, the prevalence of HIV serodiscordancy was 10.9\% (95\% confidence interval (CI) 10.2 - 11.5) overall, ranging from $6.7 \%$ (95\% CI 5.6 - 7.9) in Nsanje to $15.8 \%$ (95\% CI 14.5 - 17.3) in Ndhiwa. Men were the HIV-positive partner in $62.7 \%$ of the serodiscordant couples in Ndhiwa, in $60.4 \%$ in Gutu, in $48.8 \%$ in Chiradzulu and in 50.9\% in Nsanje. Status awareness among HIV-positive partners in serodiscordant couples ranged from $45.4 \%$ in Ndhiwa to $70.7 \%$ in Gutu. Viral load suppression (VLS) ranged from $33.9 \%$ in Ndhiwa to $68.5 \%$ in Nsanje. VLS was similar by sex in three settings, Ndhiwa (37.8\% (men) v. $27.8 \%$ (women); $p=0.16$ ), Nsanje (60.7\% v. $76.9 \% ; p=0.21$ ) and Gutu (48.2\% v. 55.6\%; $p=0.63$ ), and dissimilar by sex in Chiradzulu ( $44.4 \%$ v. $62.7 \% ; p=0.03$ ).

Conclusions. Low HIV status awareness and poor VLS among HIV-positive partners are major gaps in preventing transmission among serodiscordant couples. Intensifying programmes that target couples to test for HIV and timely antiretroviral therapy initiation could increase VLS and reduce HIV transmission.

S Afr Med J 2021;111(8):768-776. https://doi.org/10.7196/SAMJ.2021.v111i8.15489

HIV-negative individuals in a serodiscordant relationship are at high risk of HIV transmission. This risk of infection may increase if: (i) the partner living with HIV is not aware of their HIV status; ${ }^{[1,2]}(i i)$ the partner living with HIV is not on antiretroviral therapy (ART), and therefore has an unsuppressed viral load (VL); and (iii) the couple is practising unsafe sex. Serodiscordant couples are thought to be a major source of HIV transmission in the sub-Saharan Africa region, ${ }^{[3]}$ with studies estimating their contribution to be $\sim 30 \%$ of all new infections occurring in this region. ${ }^{[4,5]}$

There has recently been increasing evidence that the risk of HIV transmission to HIV-negative partners decreases when the positive partner is on ART. ${ }^{[6-15]}$ A systematic review and meta-analysis ${ }^{[9]}$ that assessed the risk of HIV transmission through unprotected sex according to VL and ART status, using data from 11 cohort studies between 1996 and 2009, found zero transmission among serodiscordant couples where the positive partner was on ART with a VL $<400$ copies/mL. In addition, other studies have shown the benefits of high ART coverage and VL suppression (VLS) in reducing the risk of transmission. ${ }^{[11,12,16]}$ Results from the HIV Prevention Trials
Network 052 trial showed that the risk of sexual transmission to the negative partner is greatly reduced if treatment is started early. ${ }^{[6,8]}$ In 2012, the World Health Organization (WHO) released guidelines recommending that positive partners in discordant couples be started on ART regardless of CD4 cell count, ${ }^{[17]}$ and the WHO 2015 guidelines $^{[18]}$ recommending universal ART irrespective of disease severity were at least partly aimed at reducing HIV transmission by achieving VLS in all people living with HIV (PLHIV).

\section{Objectives}

While there have been many studies quantifying the prevalence of serodiscordant couples, a limited number of publications have assessed the HIV cascade of care of PLHIV in serodiscordant relationships, especially in African countries and in the context of universal ART eligibility. Using population-level survey data, we quantified the prevalence of heterosexual HIV serodiscordant couples and evaluated the HIV cascade of care of PLHIV in serodiscordant relationships in four high HIV prevalence settings in sub-Saharan Africa to identify gaps in the cascade of care for this group. 


\section{Methods}

Four HIV prevalence surveys were conducted: in Ndhiwa (Kenya) in 2012, in Chiradzulu (Malawi) in 2013, and in Gutu (Zimbabwe) and Nsanje (Malawi) in 2016

\section{Study design}

We used data from four population-based HIV surveys conducted in three countries. The first two surveys were conducted in Ndhiwa (Kenya) between September and November 2012 and in Chiradzulu (Malawi) between February and May 2013. The other two surveys were more recent, conducted in Gutu (Zimbabwe) and Nsanje (Malawi), both between September and December 2016. All four surveys used similar study designs, which have been described elsewhere. ${ }^{[19-21]}$

Briefly, a two-stage sampling design was used. In the first stage, we used systematic sampling (probability proportion to size) to select clusters that followed demarcations of enumeration areas of national population and housing censuses conducted in each country. In the second stage, we randomly selected an equal number of households in each of the selected clusters ( 25 households from each cluster for all surveys except the Ndhiwa survey, which had 20 households), making the samples self-weighting. For all four surveys, we selected more than the required number of households to be used as replacements in case the dwelling unit was not found, had been destroyed or had not been inhabited for $>3$ months. All individuals aged 15 - 59 years who were residents in the study area were eligible for analysis.

At the time of the first two surveys, ART eligibility for PLHIV was a CD4 count $\leq 350$ cells $/ \mu \mathrm{L}$ or WHO stage 3 or 4 disease. In Kenya, prevention of mother-to-child transmission (PMTCT) guidelines followed WHO Option A (ART initiation in pregnant and breastfeeding women when the CD4 count is $\leq 350 / \mu \mathrm{L}$ and antiretroviral prophylaxis to prevent HIV transmission to the child if the CD4 count is $>350 / \mu \mathrm{L}$ ), while Malawi followed Option B+ (lifelong ART for all pregnant and breastfeeding women living with HIV). In 2012 and 2013, neither Kenya nor Malawi had focused on couple HIV testing and counselling as an HIV-preventive strategy, but they were beginning to implement the WHO recommendation on testing couples to enable them to make informed decisions about HIV prevention. ${ }^{[17]}$ For the 2016 surveys, Malawi and Zimbabwe had both implemented universal ART for all PLHIV - 'treat all'.

\section{Data collection methods}

In the four settings, eligible individuals who consented to take part in the survey were interviewed in their households using a structured questionnaire that included sections on demographic information, HIV testing history and ART intake for individuals who had tested HIV-positive. A rapid HIV test using the Determine Rapid kit (Abbott Laboratories, USA) was conducted if consent for HIV testing was given, the Unigold Rapid HIV test (Trinity Biotech PLC, Ireland) being used to confirm positive results and enzymelinked immunosorbent assay (Genetic Systems HIV-1/HIV-2 Plus O EIA; Bio-Rad, USA) (Western Blot in Nsanje; Bio-Rad, USA) for indeterminate rapid HIV test results. Individuals who tested HIVpositive or had indeterminate results were asked to provide a venous blood sample, which was used to conduct CD4 counts, VL and ART blood level tests (for the later surveys, an ART blood level test was done only in those who reported not being on ART). Furthermore, in the Nsanje and Gutu surveys only, we collected participants' sexual behaviour history for the 12 months prior to the survey. This information was collected for up to three most recent sexual partners, beginning with the most recent.
To ensure confidentiality, all interviews were conducted in private spaces away from other members of the household, so that when one partner was being interviewed, the other partner could not hear the interview. The collected data were double-entered and verified using EpiData 3.1 (EpiData Association, Denmark) to minimise data entry errors, and cleaned and analysed using Stata 15.0 (StataCorp, USA).

\section{Statistical methods and analysis}

We defined a stable couple as a man and a woman who reported being married or cohabiting and were living together in the same household at the time of the survey, and one member of the couple was identified as a head of the household. We divided the couples into three groups: (i) seroconcordant HIV-positive couples, where both partners were HIV-positive; (ii) HIV-serodiscordant couples, where one partner was HIV-positive and the other HIV-negative; and (iii) seroconcordant HIV-negative couples, where both partners were HIV-negative. We calculated the overall proportion of discordancy among all couples. In addition, we calculated country-specific proportions of serodiscordancy by sex to highlight the sex that contributed most to seropositivity among serodiscordant couples.

The risk of contracting HIV increases with high-risk sexual behaviour such as having unprotected sex with a person living with HIV or having multiple sexual partners. To find out whether the individuals in serodiscordant couples were engaged in high-risk sexual behaviours or not, we estimated the proportion of those who reported using a condom during the last sexual encounter and how often they used a condom with their partner during sex. We also assessed the proportion of these individuals with more than one sexual partner. Among those with sexual behaviour information, we measured the proportion of individuals who were aware of their HIV-positive status and assessed their condom use. We then verified sexual behaviour information, condom use information and how often condoms were used by couples by comparing information reported by partners to see if it matched.

We evaluated the HIV cascade of care among PLHIV in discordant relationships. Only those individuals with complete information on the variables required for calculation of the cascade of care analysis were included. The $\chi^{2}$ test was used to compare gaps in the cascade by sex. Because of the time gap between the surveys, cascade of care results were divided into earlier and later surveys for overall and survey-specific results.

\section{Ethical considerations}

Ethics approval for the studies was obtained from both local and international research ethics committees. For the two Malawi surveys, local approval was obtained from the National Health Sciences Research Committee, ref. nos 1085 (Chiradzulu) and 1598 (Nsanje). The Ndhiwa study was approved by the Kenya Medical Research Institute Ethical Review Committee (ref. no. 347) and the Gutu study by the Medical Research Council of Zimbabwe (ref. no. MRCZ/A/2075). International approval was obtained from the Comité de Protection des Personnes d'Ile de France (ref. nos 12056 (Ndhiwa) and 12084 (Chiradzulu)) and from the Médecins Sans Frontières ethics review board (ref. nos 1622 (Nsanje) and 1619 (Gutu)).

\section{Results}

Of the 21104 individuals included, 8770 (41.6\%) were living with a partner, corresponding to 4385 couples. Six individuals were in polygamous relationships and were not included as couples for this analysis. Overall, there were 952 (10.9\%) individuals (476 couples) in serodiscordant relationships, 1310 (14.9\%) individuals (655 couples) in HIV-positive concordant relationships, and 6508 (74.2\%) 
individuals (3 254 couples) with both partners HIV-negative. HIV serodiscordancy ranged from $15.8 \%$ (95\% confidence interval (CI) 14.5 - 17.3) in Ndhiwa to $10.0 \%$ (95\% CI 9.0 - 11.0) in Chiradzulu, $8.8 \%(95 \%$ CI $7.3-10.6)$ in Gutu and $6.7 \%$ (95\% CI 5.6 - 7.9) in Nsanje. Of the 476 HIV-positive partners in serodiscordant relationships, 437 (91.8\%) had complete information for evaluating the HIV cascade of care.

We found that more men than women were the HIV-positive partners in 126/201 (62.7\%) of serodiscordant couples in Ndhiwa and 29/48 (60.4\%) in Gutu, but the proportions of HIV-positive men and women were similar in serodiscordant couples in the two Malawi surveys (men 82/168 (48.8\%) in Chiradzulu and 30/59 (50.9\%) in Nsanje) (Fig. 1). Across all couple groups in all the surveys, HIV serodiscordant, seroconcordant HIV-positive and seroconcordant HIV-negative couples, there were more men in the older age groups, and men were more highly educated than women in three surveys, Ndhiwa and both Malawi sites. In Gutu, men and women had the same level of education across all couple groups. In all four surveys, a higher proportion of women than men did not report any income-generating activity, and this was similar across all HIV couple groups (Table 1).

Of 107 HIV-positive partners in serodiscordant relationships, 98 (91.6\%) had sexual behaviour data available (Nsanje and Gutu). Of these, 35 (35.7\%) used a condom during their last sexual intercourse with the sexual partner they were living with (who was their most recent sexual partner), and condom use was similar by sex (Table 2). Among those 35 individuals, 22 (62.9\%) reported always using a condom during sexual intercourse, while $11(31.4 \%)$ reported using a condom sometimes and 2 had never used a condom except for the last time they had sex. Overall, condom use was lower among serodiscordant couples (30.5\% (95\% CI 24.5 - 37.2)) than among seroconcordant positive couples $(50.7 \%$ (95\% CI $45.3-56.3))$, but higher than in seroconcordant negative couples (5.4\% (95\% CI 4.6 - 6.5)). We also found that $20.0 \%$ of positive partners in serodiscordant relationships had multiple sexual partners.

Among 97 individuals in serodiscordant relationships with sexual behaviour and HIV status awareness information available, $69.1 \%$ were aware of their HIV-positive status and 34 (50.8\%) of those who were aware used a condom at last sexual intercourse. Only $1 / 30(3.3 \%)$ of those who were unaware of their positive status used a condom. Almost all participants who were aware of their status were on ART ( $n=64 / 67 ; 95.5 \%)$, and of those on ART, 51/61 (83.6\%) had VLS and 3 had missing VL results. No difference in condom use was observed between those with VLS ( $n=25 / 51$; $49.0 \%)$ and without VLS ( $n=7 / 10 ; 70.0 \%)(p=0.23)$, probably because of low numbers.

We verified the reported information on condom use between partners in serodiscordant couples.

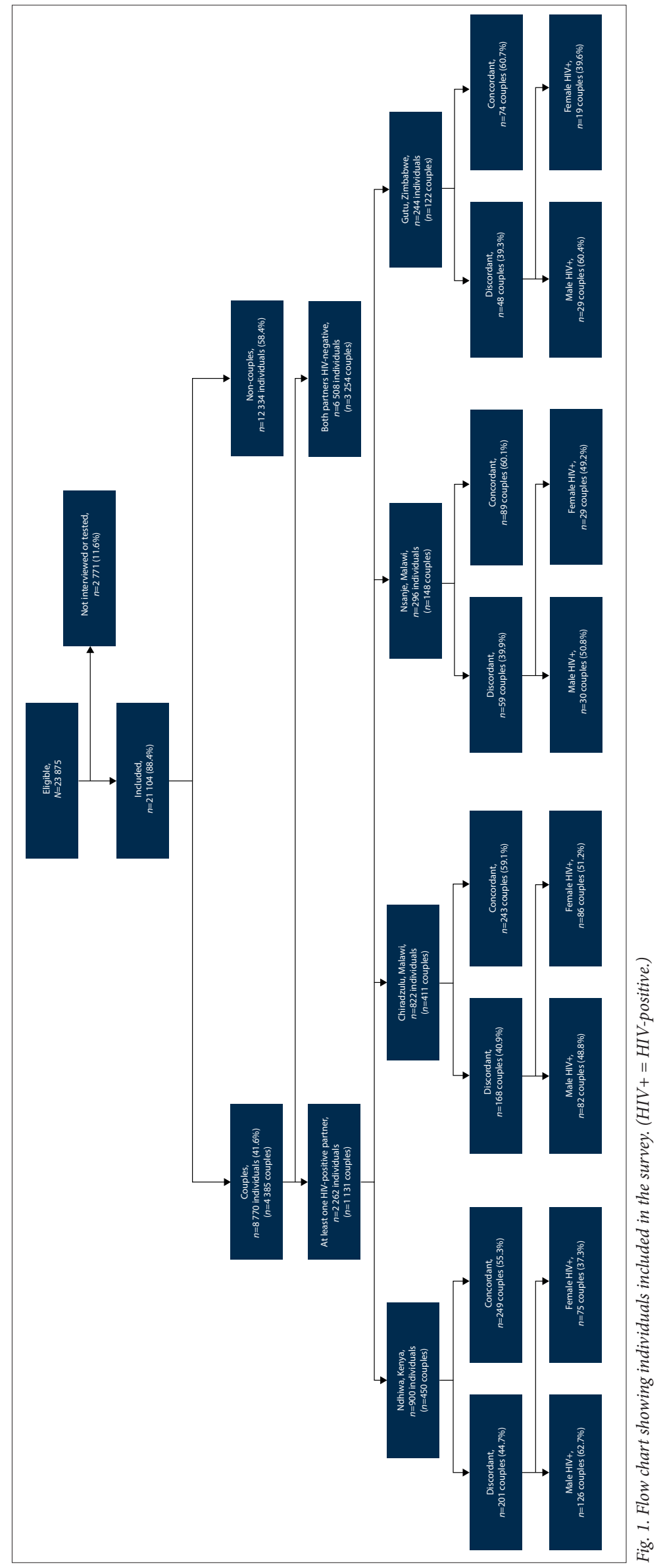




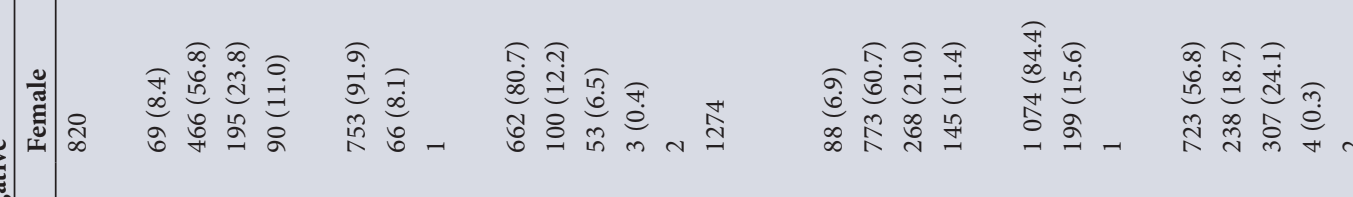

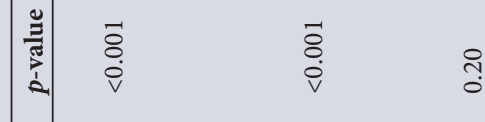

$\begin{array}{lll}\overrightarrow{0} & 0 & \overrightarrow{0} \\ \dot{0} & \stackrel{0}{v}\end{array}$

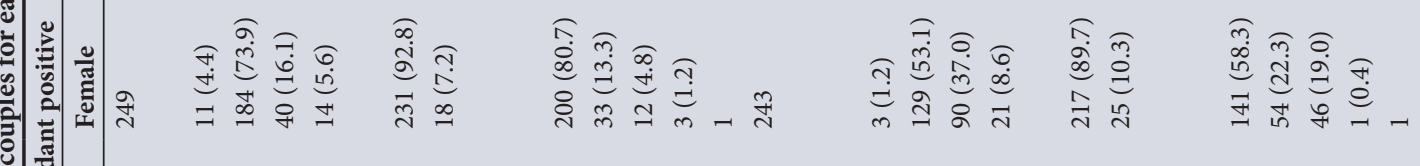

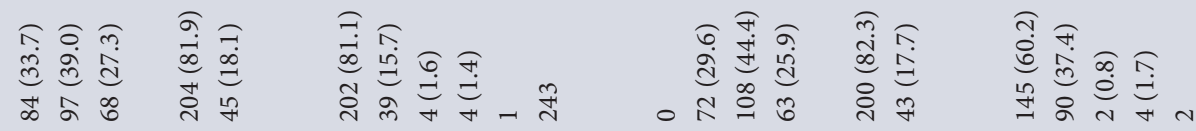

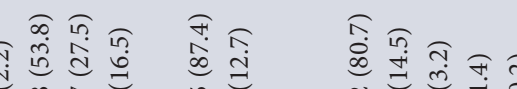

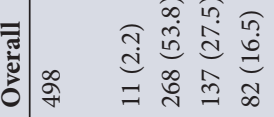
\%

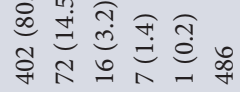

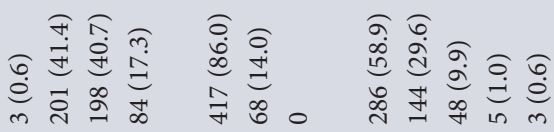

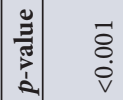

$\begin{array}{ll}\overrightarrow{8} & \overrightarrow{0} \\ \dot{0} & 0\end{array}$

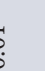

$\overrightarrow{8}$

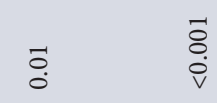

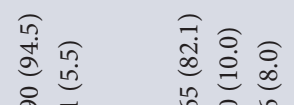

苟

苞

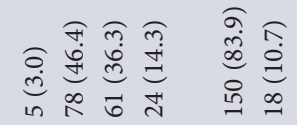

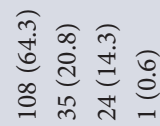

这

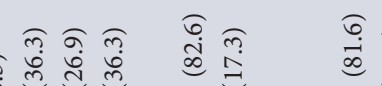

क्ष

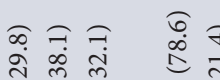

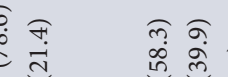

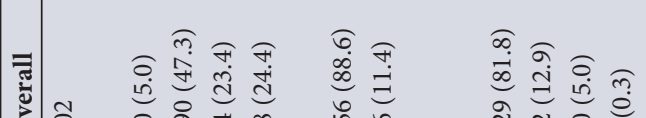

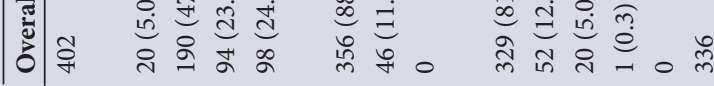

- in

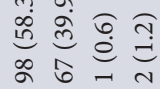

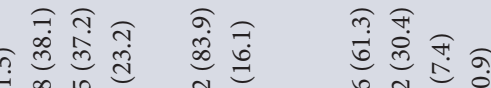

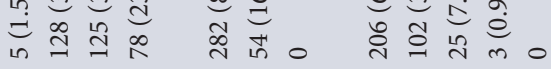

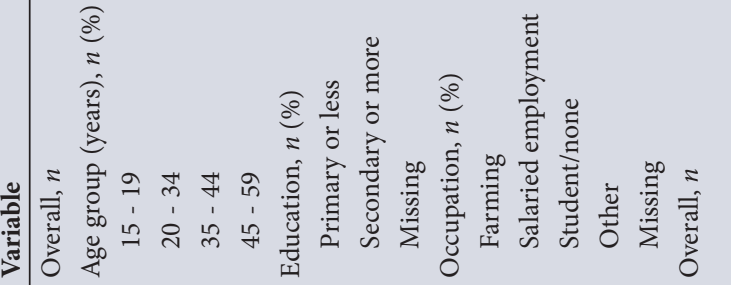

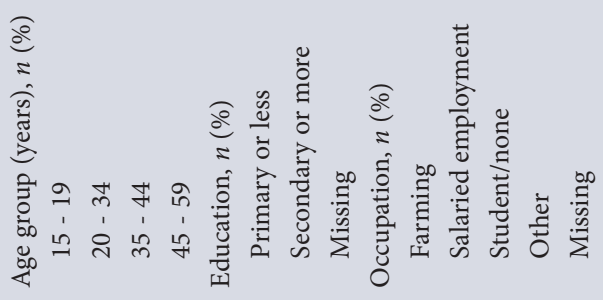

点 


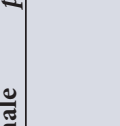

$\begin{array}{lll}\vec{\delta} & \overrightarrow{0} \\ \dot{0} & \stackrel{v}{v}\end{array}$

$\vec{\Xi}$

$\stackrel{\overrightarrow{0}}{\stackrel{0}{0}}$

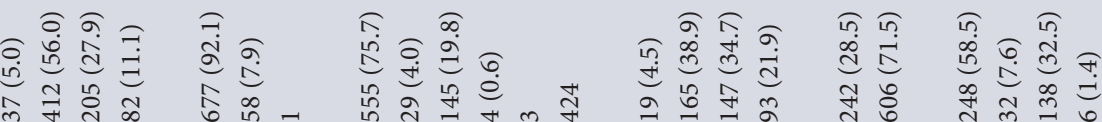

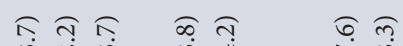

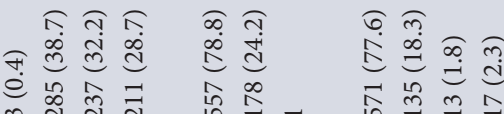

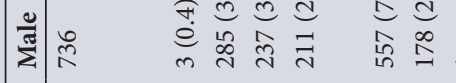

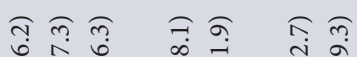

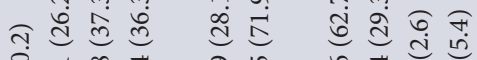

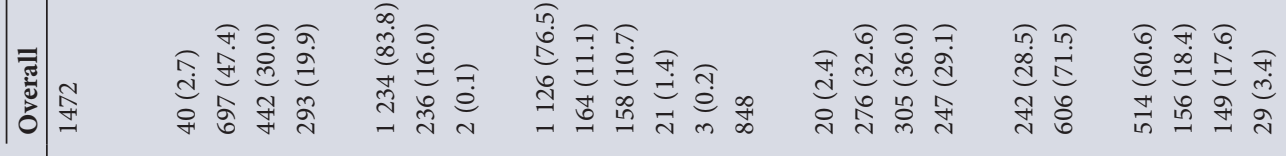

范

$\begin{array}{ll}\vec{s} & \overrightarrow{8} \\ \dot{v} & \dot{v}\end{array}$

$\begin{array}{ll}\overrightarrow{5} & \overline{0} \\ \dot{0} & \end{array}$

$\stackrel{\infty}{0} \quad \stackrel{\overline{0}}{\dot{0}}$

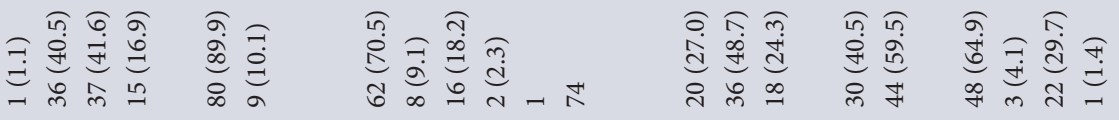

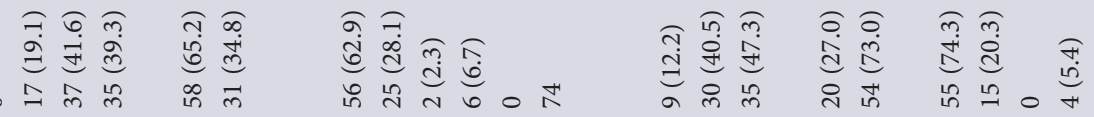

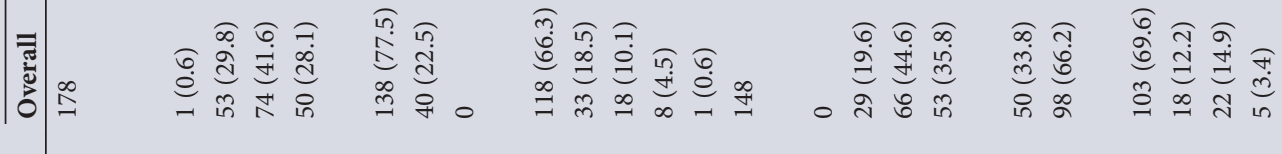

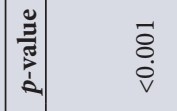

$\begin{array}{lllll}\overrightarrow{0} & 0 & 0 & 0 \\ 0 & 0 & 0 & 0 \\ 0 & 0 & 0\end{array}$

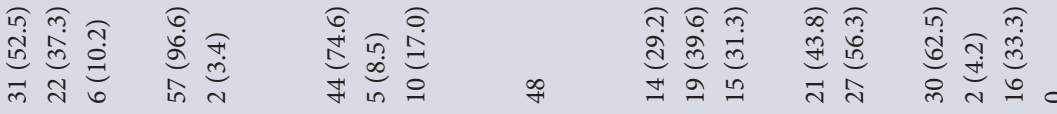

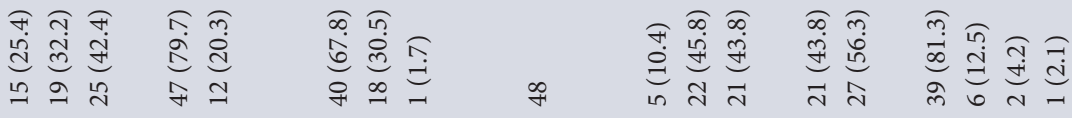

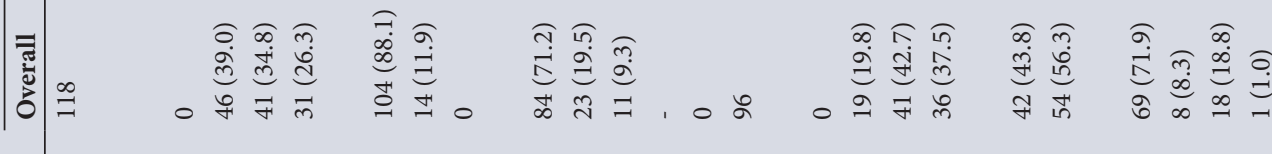

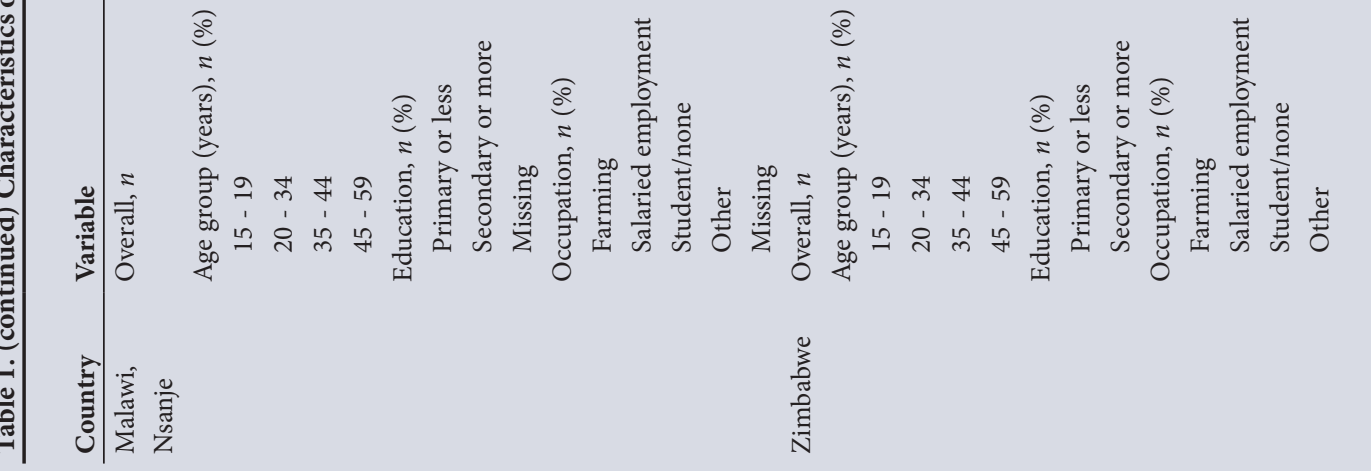




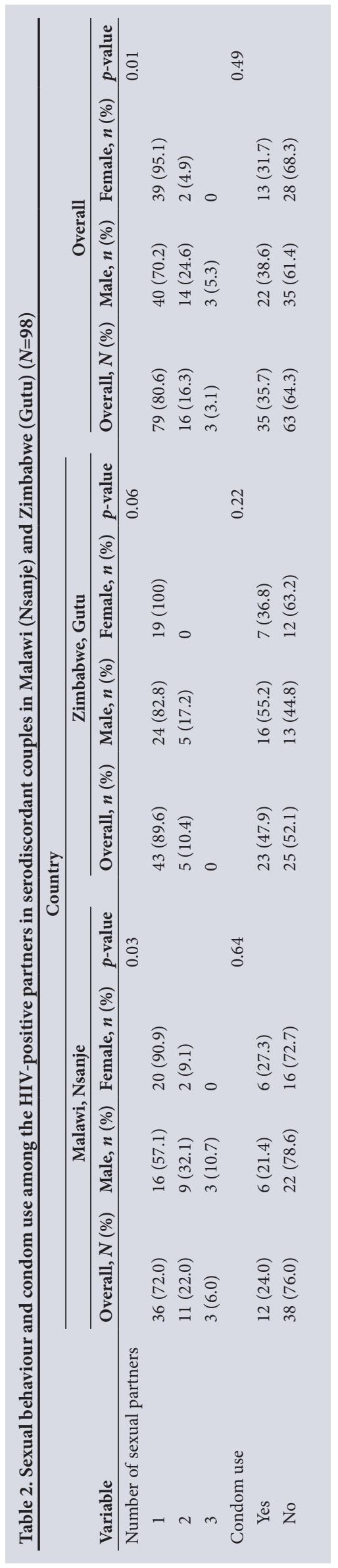

Of the 107 serodiscordant couples, 78 (72.9\%) gave matching information about condom use, 19 (17.8\%) gave mismatched or discrepant information, and $10(9.4 \%)$ had missing information. Of the 78 couples with matching condom use information, $20(25.6 \%)$ gave matching information that they had used a condom in the last sexual encounter; of these, 18/20 (90.0\%) gave matching information on how often they used a condom. Among those who were aware of their HIV-positive status, 48/67 (71.6\%) gave matching information with their partner regarding condom use, and 29/48 $(60.4 \%)$ of these gave matching information on not using a condom. However, almost all respondents with matching information on not using condoms were on ART (96.5\%), and $92.3 \%$ of these had VLS.

Thirty-nine PLHIV were missing VL data. Of these, 6 (15.4\%) were aware of their status, $5(12.8 \%)$ were in care and $5(12.8 \%)$ were on ART. For the first two surveys (Ndhiwa and Chiradzulu), overall, of the 238 HIV-positive partners in serodiscordant relationships with complete data for the HIV cascade of care, $55.3 \%$ were aware of their status, $50.9 \%$ were linked to care, $49.4 \%$ were still in care, $41.4 \%$ were on ART and $43.2 \%$ had VLS. Gaps in the first three stages of the cascade of care (HIV status awareness, linkage to care and retention in care) were smaller for women than for men, but there was no difference by gender in the last two stages (on ART and VLS). Site-specific results showed smaller gaps in almost all stages of the cascade of care in Chiradzulu compared with Ndhiwa. Gaps were smaller among Chiradzulu women v. men in all cascade of care stages, while no sex differences were observed in Ndhiwa (Fig. 2).

For the later surveys in Gutu and Nsanje, of the 99 HIV-positive partners with complete HIV cascade of care information, $70.7 \%$ were aware of their HIV status, $69.7 \%$ were linked to care, $68.7 \%$ were still in care and on ART and $60.6 \%$ had VLS. We found no differences by sex in any stage of the cascade of care. Site-specific results were also similar in all stages of the cascade of care. However, cascade of care gaps were smaller in women in Nsanje in all stages except VLS, while there were no differences in any stage in Gutu (Fig. 3).

Gaps in the HIV care cascade were greater in PLHIV in discordant couples v. the overall population of PLHIV (awareness of HIV status in PLHIV in discordant couples v. the general population was $45.4 \%$ (95\% CI 38.3 - 52.6) v. $59.4 \%$ (95\% CI 56.8 - 61.9) in Ndhiwa, ${ }^{[20]} 67.1 \%(95 \%$ CI $59.3-74.1)$ v.
$77.0 \%$ (95\% CI 74.4 - 79.3) in Chiradzulu, ${ }^{[19]}$ $70.4 \%$ (95\% CI 56.9 - 81.0) v. $80.0 \%(95 \%$ CI 76.4 - 83.1) in Nsanje, and $71.1 \%(95 \%$ CI 56.3 - 82.5) v. $87.4 \%$ (95\% CI 84.7 89.8) in Gutu ${ }^{[21]}$ ). VLS among HIV-positive partners in discordant couples v. the general population was $33.9 \%$ (95\% CI 27.4 - 41.1) v. $39.7 \%\left(95 \%\right.$ CI 37.1 - 42.4) in Ndhiwa, ${ }^{[20]}$ $54.2 \%$ (95\% CI 46.3 - 61.9) v. $61.9 \%$ (95\% CI 58.9 - 64.7) in Chiradzulu, ${ }^{[19]} 68.5 \%$ (95\% CI 55.0 - 79.5) v. $73.1 \%$ (95\% CI 69.2 - 76.7) in Nsanje, and $51.1 \%$ (95\% CI 36.8 - 65.3) v. $71.6 \%$ (95\% CI 68.0 - 75.0) in Gutu. ${ }^{[21]}$

\section{Discussion}

This study is the first multi-country population-based survey to quantify HIV serodiscordancy and the cascade of care of PLHIV in serodiscordant relationships in sub-Saharan Africa. We found a high prevalence of HIV serodiscordant couples, with men most likely to be the HIV-positive partners in the study sites in Ndhiwa and Gutu. Coverage of steps in the HIV cascade of care for serodiscordant couples was generally low, with little difference between men and women. The majority of serodiscordant couples were still practising high-risk sexual behaviours. Owing to low cascade of care outcomes and ongoing sexual risk behaviour, serodiscordant couples may remain a major source of HIV transmission.

The prevalence of serodiscordancy was high in every site. Our population estimates of the prevalence of serodiscordant couples are different to the national estimates. In Kenya, a study in 2012 estimated the prevalence of HIV serodiscordant couples at the national level among 15 - 64-year-olds to be $\sim 5 \%,{ }^{[22]}$ and between 2008 and 2009, this prevalence was estimated to be $\sim 6 \%$ among 15 - 54-year-olds using Demographic Health Survey (DHS) data. ${ }^{[23]}$ In Malawi, our estimate in the Chiradzulu study was also relatively higher than a 2010 national estimate from a study that looked at trends in prevalence of HIV discordancy, which found that $\sim 8 \%$ of couples aged $15-54$ years were in serodiscordant relationships, and the proportion did not change between 2015 and 2016. ${ }^{[23]}$ In Zimbabwe, however, the proportion of serodiscordant couples in our study was similar to the national 2015 estimates among couples aged 15 - 54 years. ${ }^{[23]}$ The big difference between our results and the Kenya national estimates could be explained in part by the difference in HIV prevalence between the area of the study (25\%) and the national prevalence estimates ( $6 \%$ in 2012). ${ }^{[22]}$ Geographical location plays a key role in the distribution 


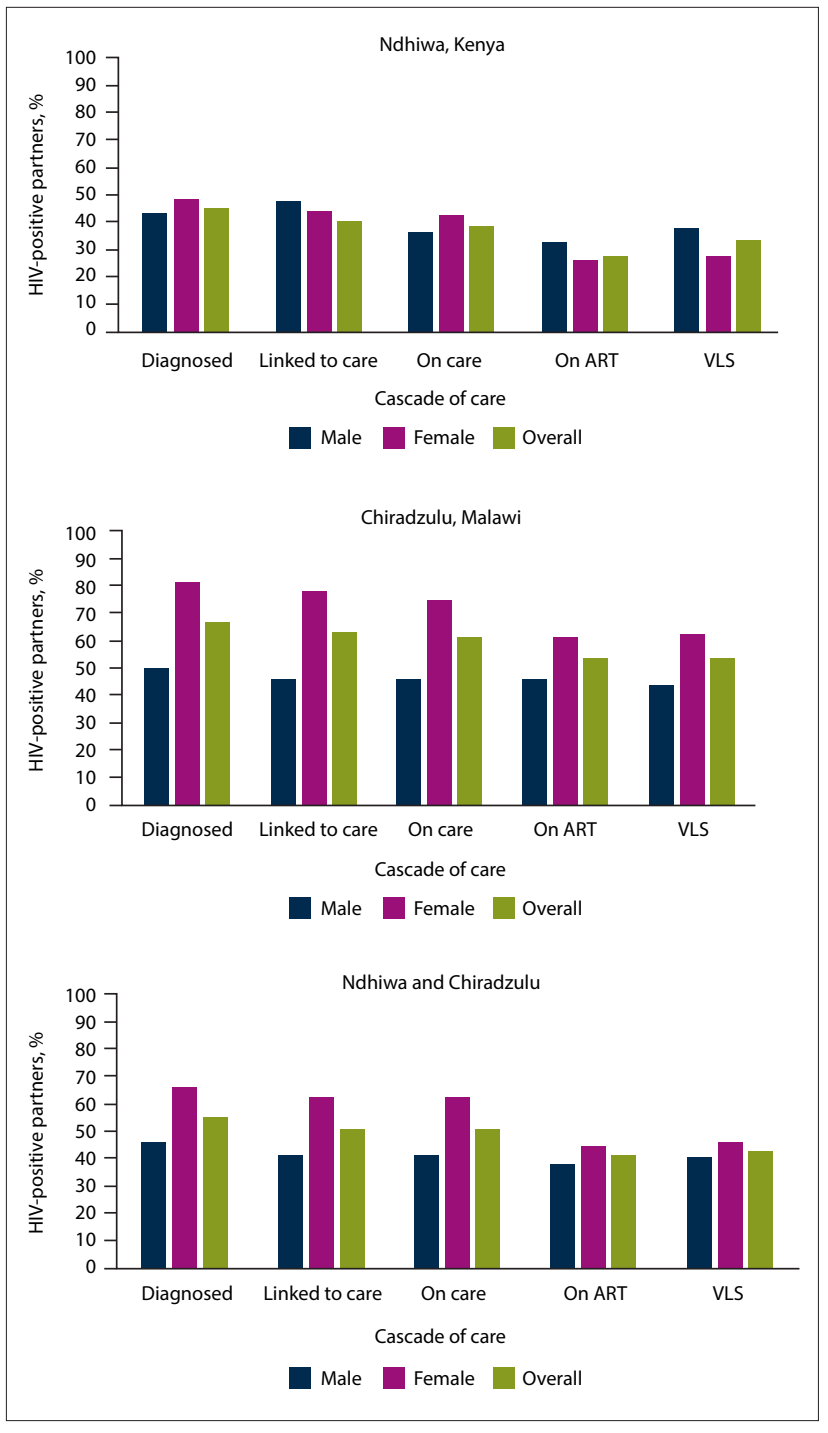

Fig. 2. Cascade of care among 238 HIV-positive partners in discordant couples in Kenya (Ndhiwa) and Malawi (Chiradzulu). (ART = antiretroviral therapy; VLS = viral load suppression.)

of HIV in Kenya; in some areas, HIV prevalence is as low as $0.4 \%$, while in other areas it can be as high as $26 \%{ }^{[24]}$ Similar to our results, except for the two Malawi surveys, other studies have found men to comprise a higher proportion of positive partners in serodiscordant relationships. ${ }^{[23]}$

There have been few studies examining the HIV cascade of care of PLHIV in serodiscordant relationships. ${ }^{[25]}$ We found generally larger gaps in the cascade of care outcomes among HIV-positive partners in serodiscordant relationships $\mathrm{v}$. the general population in all three countries. This may mean that specific interventions targeting PLHIV in discordant relationships are required or should be intensified to make sure that this group achieves the same level of status awareness and VLS as the general population. Moreover, the proportion of $\sim 40 \%$ of PLHIV in serodiscordant relationships with a VL $\geq 1000$ copies/mL in all four settings is high and could be a major source of HIV transmission within couples. This risk is exacerbated if individuals are unaware of their status, as they are less likely to be practising preventive measures. ${ }^{[1,2]}$ We also found that in the first surveys, the cascade of care coverage for Ndhiwa was lower compared with Chiradzulu. Among the later surveys, the cascade

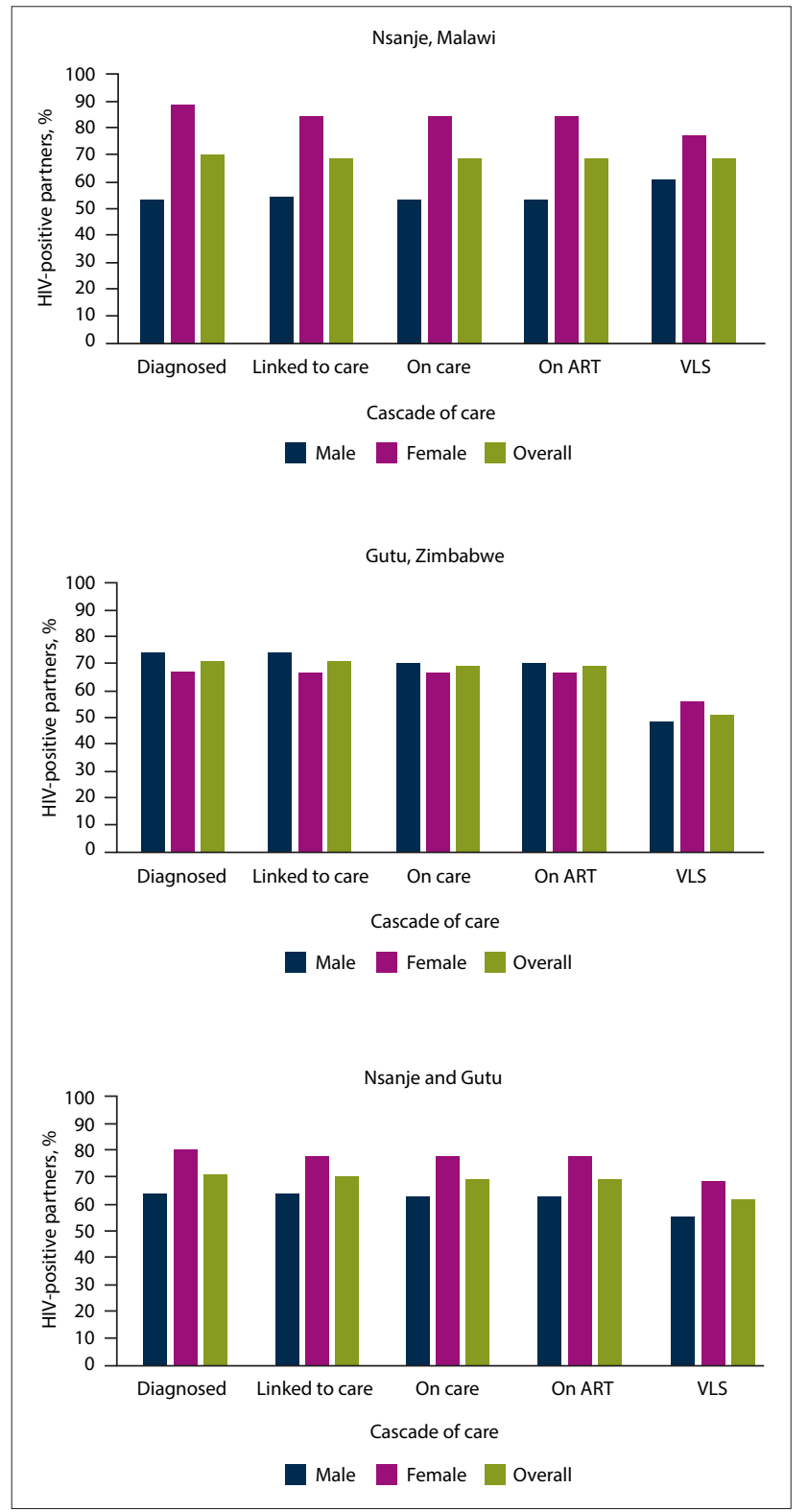

Fig. 3. Cascade of care among $99 \mathrm{HIV}$-positive partners in discordant couples in Malawi (Nsanje) and Zimbabwe (Gutu). (ART = antiretroviral therapy; VLS = viral load suppression.)

of care coverage for Nsanje and Gutu was similar in all cascade of care stages. We observed a substantial improvement in coverage of almost all stages of the HIV cascade of care when comparing the first and later surveys, probably because in recent years these countries have become proactive in implementing methods to increase testing coverage, linking PLHIV to care and starting ART with adoption of the WHO 'treat all' recommendation. ${ }^{[18]}$ As a deliberate measure, Kenya implemented specific policies aimed at increasing HIV status awareness among couples in response to the large number of HIV serodiscordant couples (estimated at 260000 nationwide in 2012). ${ }^{[2]}$

Overall, in the first surveys in Ndhiwa and Chiradzulu, women were more likely than men to know their HIV status and be retained in care, but the proportions of individuals on ART and virally suppressed were similar. In the later surveys in Gutu and Nsanje, women and men were similar in all steps of the cascade of care. In both Malawi surveys, women had better outcomes than men in all 
steps of the cascade of care, except for VLS in Nsanje, where women and men were similar. Malawi's very proactive PMTCT programme with early adoption of Option $\mathrm{B}+$ is probably the reason for these sex differences. These findings also reinforce the view that diagnosis in men remains key to controlling the HIV epidemic and that testing and counselling in couples should be promoted because it encourages preventive behaviour in couples, thus reducing the risk of transmission. ${ }^{[26,27]}$

Evidence from the literature suggests that men are less likely to access ART than women, and that in some countries sex differences in ART coverage are increasing. ${ }^{[28]}$ This is to be expected, given that some of the services related to HIV status awareness and ART are readily available to women as part of routine antenatal care services, but not to men, and ART eligibility criteria in several countries were less restrictive for pregnant and breastfeeding women as part of PMTCT. Women in discordant relationships are therefore also expected to have better outcomes than men in all steps of the HIV care cascade. In order to increase status awareness and ART coverage among men, who represent the highest proportion of PLHIV among serodiscordant couples, other studies have suggested introducing routine HIV testing services similar to those accessed by women in antenatal clinics. ${ }^{[28]}$ In addition, implementation of the 'treat all' guidelines that most countries have already adopted and implemented, community-based approaches to HIV testing and ensuring that men stay on treatment might also help to reduce the existing HIV cascade gap between men and women, specifically for Malawi.

Our results generally show low condom use among PLHIV in serodiscordant couples, similar to findings of other studies. ${ }^{[1,22]}$ When we looked at condom use according to HIV status awareness, we found that condom use was low among individuals who were aware of their status, but that it was higher than among those who were not aware of their status. However, it is encouraging to note that the majority of those who were aware of their status were on ART and had VLS, and that in general, of those who reported using a condom in their last sexual encounter, most always used a condom.

The fact that almost all the individuals who reported using a condom were aware of their HIV-positive status adds weight to the importance of knowing one's status. Verifying the information given on condom use improved the credibility of our data, as the agreement between partners was very good. However, we found that in 30\% of those who were aware of their HIV-positive status, their reported use of a condom at their last sexual encounter was not consistent with what their partners reported. It is difficult to ascertain which partner was reporting accurately, but if these results reflect absence of condom use, it would further reduce the proportion of those using condoms, overall and among those who were aware of their status.

\section{Study limitations}

Our study has several limitations. No polygamous relationships were included, as the study was not designed to identify polygamous families, which may underestimate the prevalence of serodiscordant couples, especially in areas where polygamy is prevalent. In addition, because we only included couples where one member was the head of the household, some individuals who were still living in the same household as their parents were not included as couples, which could also lead to underestimation of prevalence of serodiscordant couples. Obtaining and ascertaining accurate sexual behaviour data is difficult, with men tending to report more sexual partners than women. ${ }^{[2,30]}$ However, we were able to verify condom use.

\section{Conclusions}

While there has been an improvement in coverage of the cascade of care in PLHIV in serodiscordant couples between the first (Ndhiwa and Chiradzulu) and later surveys (Gutu and Nsanje), the high prevalence of serodiscordant couples with low status awareness among HIV-positive partners, even in the later surveys, is a major gap in HIV care and prevention. The low prevalence of status awareness among HIV-positive partners must be addressed in order to promote timely ART initiation and/or pre-exposure prophylaxis to reduce transmission. We also recommend repeating the surveys in the same areas, targeting the same population to assess changes over time in the prevalence and the cascade of care of serodiscordant couples.

Declaration. The research for this study was done in partial fulfilment of the requirements for MLC's DPhil (Public Health) degree at the University of Cape Town.

Acknowledgements. The following are acknowledged as contributing to the success of the study: the study participants, survey field teams and MSF staff, and all the individuals who contributed towards the success of the surveys in all four countries. We also thank the South African Department of Science and Technology/National Research Foundation (DST-NRF) Centre of Excellence in Epidemiological Modelling and Analysis (SACEMA), Stellenbosch University, South Africa, for their support.

Author contributions. MLC: conceptualisation, data analysis and interpretation, drafting of the article and study design; NC: critical review of the article and study design; TE: critical review of the article; EP: critical review of the article and final approval; DBG: critical review of the article; RO: critical review of the article; SW: critical review of the article; $\mathrm{CM}$ : critical review of the article; J-FE: critical review of the article and study design; M-AD: conceptualisation, critical review of the article and study design; DM: conceptualisation, critical review and study design.

Funding. None.

Conflicts of interest. None.

1. Huerga H, Venables E, Ben-Farhat J, van Cutsem G, Ellman T, Kenyon C. Higher risk sexual behaviour is associated with unawareness of HIV-positivity and lack of viral suppression - implications for treatment as prevention. Sci Rep 2017;7:16117. https://doi.org/10.1038/s41598-017-16382-6

2. National AIDS Control Council, Kenya. Kenya AIDS Strategic Framework 2014/2015 - 2018/2019. NACC, 2015, https://nacc. orke/wp-content/uploads/2015/09/KASF_Final pdf (accessed 3 November 2020).

3. Chemaitelly H, Awad SF, Abu-Raddad LJ. The risk of HIV transmission within HIV-1 sero-discordant couples appears to vary across sub-Saharan Africa. Epidemics 2014;6:1-9. https://doi.org/10.1016/j. couples appears to

4. Chemaitelly H, Shelton JD, Hallett TB, Abu-Raddad LJ. Only a fraction of new HIV infections occur Chemaitelly H, Shelton JD, Hallett TB, Abu-Raddad LJ. Only a fraction of new HIV infections occur
within identifiable stable discordant couples in sub-Saharan Africa. AIDS 2013:27(2):251-260. https:// within identifiable stable discordant couples in sub-Saharan Africa. AIDS 2013;27(2):251-260. https://
doi.org/10.1097/QAD.0b013e32835ad459

5. Chemaitelly H, Awad SF, Shelton JD, Abu-Raddad LJ. Sources of HIV incidence among stable couples in sub-Saharan Africa. J Int AIDS Soc 2014;17(1):18765. https://doi.org/10.7448/IAS.17.1.18765

6. Cohen MS, Chen YQ, McCauley M, et al. Prevention of HIV-1 infection with early antiretroviral therapy. N Engl J Med 2011;365(6):493-505. https://doi.org/10.1056/NEJMoa1 105243

. Cohen MS, Smith MK, Muessig KE, Hallett TB, Powers KA, Kashuba AD. Antiretroviral treatment of HIV-1 prevents transmission of HIV-1: Where do we go from here? Lancet 2013;382(9903):1515-1524. https://doi.org/10.1016/S0140-6736(13)61998-4

8. Cohen MS, Chen YQ McCauley M, et al. Antiretroviral therapy for the prevention of HIV-1 transmission. N Engl J Med 2016;375(9):830-839. https://doi.org/10.1056/NEJMoal600693

9. Attia S, Egger M, Müller M, Zwahlen M, Low N. Sexual transmission of HIV according to viral load Attia S, Egger M, Muller M, Zwahlen M, Low N. Sexual transmission of HIV according to viral load
and antiretroviral therapy: Systematic review and meta-analysis. AIDS 2009;23(11):1397-1404. https:// and antiretroviral therapy: Systematic review

10. Bavinton BR, Pinto AN, Phanuphak N, et al. Viral suppression and HIV transmission in serodiscordant male couples: An international, prospective, observational, cohort study. Lancet HIV serodiscordant male couples: An international, prospective, observational, cohort study. Lancet HIV 2018;5(8):e438-e447. https://doi.org/10.1016/s2352-3018(18)30132-2

11. Tanser F, Bärnighausen T, Grapsa E, Zaidi J, Newell ML. High coverage of ART associated with decline in risk of HIV acquisition in rural KwaZulu-Natal, South Africa. Science 2013;339(6122):966-971. https://doi.org/10.1126/science.1228160

12. Wilson DP, Law MG, Grulich AE, Cooper DA, Kaldor JM. Relation between HIV viral load and infectiousness: A model-based analysis. Lancet 2008;372(9635):314-320. https://doi.org/10.1016/ S0140-6736(08)61115-0 
13. Rodger AJ, Cambiano V, Bruun T, et al. Sexual activity without condoms and risk of HIV transmission in serodifferent couples when the HIV-positive partner is using suppressive antiretroviral therapyin serodifferent couples when the HIV-positive partner is using suppc

14. He N, Duan S, Ding Y, et al. Antiretroviral therapy reduces HIV transmission in discordant couples in rural Yunnan, China. PLoS One 2013;8(11):e77981. https://doi.org/10.1371/journal.pone.0077981 15. Jia Z, Mao Y, Zhang F, et al. Antiretroviral therapy to prevent HIV transmission in serodiscordant 5. Jia Z, Mao Y, Zhang F, et al. Antiretroviral therapy to prevent HIV transmission in serodiscordant couples in China (2003 - 11): A national observation
1203. https://doi.org/10.1016/s0140-6736(12)61898-4

16. Vandormael A, Newell ML, Bärnighausen T, Tanser F. Use of antiretroviral therapy in households and risk of HIV acquisition in rural KwaZulu-Natal, South Africa, 2004 - 12: A prospective cohort study. Lancet Glob Health 2014;2(4):e209-215. https://doi.org/10.1016/S2214-109X(14)70018-X

17. World Health Organization. Guidance on couples HIV testing and counselling including antiretroviral therapy for treatment and prevention in serodiscordant couples: Recommendations for a public health approach. Geneva: WHO, 2012. https://www.who.int/hiv/pub/guidelines/9789241501972/en/ (accessed 5 November 2020)

18. World Health Organization. Guideline on when to start antiretroviral therapy and on pre-exposure prophylaxis for HIV. Geneva: WHO, 2015. https://sahivsoc.org/Files/New\%20WHO\%20ART\%20 and\%20PrEP\%20guidelines.pdf (accessed 5 November 2020).

19. Maman D, Chilima B, Masiku C, et al. Closer to 90-90-90: The cascade of care after 10 years of ART scale-up in rural Malawi: A population study. J Int AIDS Soc 2016;19(1):20673. https://doi. org/10.7448/IAS.19.1.20673

20. Maman D, Zeh C, Mukui I, et al. Cascade of HIV care and population viral suppression in a high-burden region of Kenya. AIDS 2015;29(12):1557-1565. https://doi.org/10.1097/QAD.0000000000000741

21. Conan N, Coulborn RM, Simons E, et al. Successes and gaps in the HIV cascade of care of a high HIV prevalence setting in Zimbabwe: A population-based survey. J Int AIDS Soc 2020;23(9):e25613. https://doi.org/10.1002/jia2.25613

22. Maina WK, Kim AA, Rutherford GW, et al. Kenya AIDS Indicator Surveys 2007 and 2012: Implications for public health policies for HIV prevention and treatment. J Acquir Immune Defic Syndr 2014;66(Suppl 1):S130-S137. https://doi.org/10.1097/QAI.0000000000000123
23. Denardo RD. Prevalence of HIV-discordant couples in sub-Saharan Africa: What has changed over the last decade? 27 April 2018. https://paa.confex.com/paa/2018/meetingapp.cgi/Paper/24389 (accessed 5 November 2020).

24. National AIDS Control Council, Kenya. Kenya HIV estimates 2015. Nairobi: NACC, 2016. http://nace. or.ke/wp-content/uploads/2016/12/Kenya-HIV-Estimates-2015.pdf (accessed 5 November 2020).

25. Jonnalagadda S, Auld A, Payne D, et al. Awareness and disclosure of status and undetectable viral load in HIV-serodiscordant couples: Evidence from population-based HIV impact assessments. Presented
in at the 20th ICASA International Conference on AIDS and STIs in Africa, Kigali Convention Centre, Kigali, Rwanda, 2 - 7 December 2019. https://www. professionalabstracts.com/icasa2019/iplanner/\#/ Kigali, Rwanda, 2 - 7 December 2019. https://

26. Wall KM, Kilembe W, Vwalika B, et al. Sustained effect of couples' HIV counselling and testing on risk reduction among Zambian HIV serodiscordant couples. Sex Transm Infect 2017;93(4):259-266. https://doi.org/10.1136/sextrans-2016-052743

27. King R, Min J, Birungi J, et al. Effect of couples counselling on reported HIV risk behaviour amon HIV serodiscordant couples by ART use, HIV status and gender in rural Uganda. PLoS ONE 2015;10(9):e0136531. https://doi.org/10.1371/journal.pone.0136531

28. Auld AF, Shiraishi RW, Mbofana F, et al. Lower levels of antiretroviral therapy enrollment among men with HIV compared with women - 12 countries, 2002 - 2013. MMWR Morb Mortal Wkly Rep 2015;64(46):1281-1286. https://doi.org/10.15585/mmwr.mm6446a2

29. Mercer CH, Tanton C, Prah P, et al. Changes in sexual attitudes and lifestyles in Britain through the life course and over time: Findings from the National Surveys of Sexual Attitudes and Lifestyles (Natsal). Lancet 2013;382(9907):1781-1794. https://doi.org/10.1016/S0140-6736(13)62035-8

30. Todd J, Cremin I, McGrath N, et al. Reported number of sexual partners: Comparison of data from four African longitudinal studies. Sex Transm Infect 2009;85(Suppl 1):i72-i80. https://doi.org/10.1136/ sti.2008.033985

Accepted 23 February 2021 